Article

\title{
On the Role of Unitary-Symmetry for the Foundation of Probability and Time in a Realist Approach to Quantum Physics
}

\author{
Andreas Schlatter \\ Burghaldeweg 2F, 5024 Küttigen, Switzerland; schlatter.a@bluewin.ch; Tel.: +41-(0)-62-827-00-16
}

Received: 14 November 2018; Accepted: 6 December 2018; Published: 10 December 2018

check for updates

\begin{abstract}
We show that probabilities in quantum physics can be derived from permutation-symmetry and the principle of indifference. We then connect unitary-symmetry to the concept of "time" and define a thermal time-flow by symmetry breaking. Finally, we discuss the coexistence of quantum physics and relativity theory by making use of the thermal time-flow.
\end{abstract}

Keywords: born-rule; density operator; principle of indifference; relativity; symmetry; thermal flow; time

\section{Introduction}

Quantum theory, which originated in the early 20th century, has been defined in its mathematical structure for decades. Its empirical success has ever since been astounding. And yet, there still is a vivid debate around the interpretation of the formalism. The mathematical theory seems to be ontologically underdetermined [1]. Among the key issues, the questions range from the nature of the probabilities, which seem fundamental to the theory, to the notion of "time". A related question is the coexistence of quantum physics with relativity theory. We want to show in this paper that the concept of unitary-symmetry and symmetry-breaking by measurement can be the starting point for an understanding of these questions. The paper is structured as follows. In Section 2 we will show that symmetry under permutations of orthogonal states lies at the heart of the Born-rule. In Section 3 we will relate unitary-symmetry to the notion of change and "time" and show that the breaking of unitary-symmetry, jointly with the probabilities, leads to an empirical time-flow, which we call thermal time. Finally, in Section 4 we consider space and time and discuss the coexistence of relativity theory and quantum physics with the help of the thermal time-flow. Of course, unitary symmetry is a foundational concept of quantum theory far beyond the scope of this paper. This becomes particularly clear in quantum field theory [2].

\section{Probabilities}

Every interpretation of quantum physics today needs to introduce probabilities in order to explain experimental results, which are only available in form of statistics. Independently of quantum physics, there has for a much longer time been a debate in philosophy what probabilities really are [3]. Consequently, the various interpretations of quantum physics take different positions regarding the nature of probabilities. Given the formalism, there are mathematical proofs of the existence and uniqueness of a corresponding probability measure. The most famous result stems from Gleason [4]. Aage Bohr and O. Ulfbeck derive the probabilities directly from the symmetries of the theory [5]. The basis of these results is the axiomatic definition of probability measures. To rely on these proofs to understand the nature of probability, however, carries a flavor of circularity. The Bayesian approach is shared by QBism [6] jointly with the many-worlds interpretation [7]. 
In these interpretations probabilities are subjective degrees of belief, which allow agents to take rational decisions. (The intuition can be gained from the "Dutch-book" argument.) While somebody adopting QBism needs to explain why the world is manifesting itself probabilistically, the many-worlds interpretation definitely needs to explain why probabilities exist at all. This is not immediately clear, if all branches of the quantum state are realized [8]. The frequency approach, where ensembles of physical systems are considered, is the most operative of all, since it represents what experimentalists do in the lab. It is the underlying probability concept of the Copenhagen interpretation. Since its definition is based on ensembles, there is the inherent difficulty to interpret a single trial or single event. Based on the frequency and the Bayesian notion, there are successful derivations of quantum physics as a probabilistic theory from a few (four or five) axioms $[9,10]$. Finally, hidden variable theories, like Bohmian mechanics for instance, rely on the epistemic or ignorance approach to probability. There is a vast literature on the topic, and this short overview makes no pretense of giving an adequate account of the discussion. We take another route instead.

There is an understanding of probability, shared by a generation of scientists like Bernoulli, Leibniz, Laplace and Euler: Due to symmetry, there arises a lack of distinguishability between certain individual events and properties of a system and hence, by the principle of insufficient reason or indifference, these events or properties are ex-ante equally probable. This fundamental intuition creates a link between the world, which embodies some symmetry, and the observer, who responds by indifference to the resulting inability to distinguish. The principle of indifference, though at first sight Bayesian, has thus a basis in reality, namely in the symmetry of certain properties. By sharpening an argument in reference [11], we will show, in a first step, that this view of probability can be discovered in the formalism of quantum physics

\subsection{Quantum Physics}

The ansatz for the mathematical theory of quantum physics is to represent a measurable property of a physical system, called an observable, as a self-adjoint operator $A \in L\left(H_{\mathbb{C}}^{d}\right)$ in the space of linear operators over a state space $H_{\mathbb{C}}^{d}$, which carries the structure of a complex Hilbert space of some dimension $d \epsilon \mathbb{N}$. (We only consider the finite-dimensional case.) The values, which this property can assume in an experiment, are the corresponding eigenvalues $\lambda_{k} \in \mathbb{R}, k \leq d$, of $A$. (We assume that there are $d$ different, non-degenerate eigenvalues.) Quantum theory assigns probabilities to these eigenvalues, which are then observed in repeated experiments on identically prepared systems. To do this, states are represented as unit-vectors $|\psi\rangle \in H_{\mathbb{C}}^{d},\|\psi\|=1$. They can be linearly expanded on the basis of orthonormal eigenstates of $A,\left\{\left|e_{k}\right\rangle\right\}_{k \leq d} \subset H_{\mathbb{C}}^{d}|\psi\rangle=\sum_{k=1}^{d} \psi_{k}\left|e_{k}\right\rangle, \psi_{k} \in \mathbb{C}$. A probability $p_{k}$ is then assigned to the measurement of eigenvalue $\lambda_{k}, k \leq d$, by

$$
p_{k}=\left|\psi_{k}\right|^{2} .
$$

This assignment is also known as the "Born-rule" [12]. There is another way to represent states, which is more general. We define the (one-dimensional) orthogonal projection operators $\left.\left\{\Pi_{k}\left|\Pi_{k}=\right| e_{k}\right\rangle\left\langle e_{k}\right|\right\}_{k \leq d} \subset L\left(H_{\mathbb{C}}^{d}\right)$, projecting to the eigenspaces of $A$. Orthogonal projections, $\Pi$, are idempotent and self-adjoint, two properties which define this class of operators:

$$
\Pi^{2}=\Pi, \Pi=\Pi^{*} .
$$

A pure state is a projector $\varrho_{\psi}$, associated with a unit-vector $|\psi\rangle \epsilon H_{\mathbb{C}}^{d}$

$$
\varrho_{\psi}=|\psi\rangle\left\langle\psi\left|=\sum_{i, k=1}^{d} \psi_{i} \psi_{k}^{*}\right| e_{i}\right\rangle\left\langle e_{k}\right| .
$$


With $\operatorname{tr}: L^{2}\left(H_{\mathbb{C}}^{d}\right) \rightarrow \mathbb{C}$ denoting the trace-operator and $\Pi_{k}$ the projector to the eigenspace, spanned by $\left|e_{k}\right\rangle$, the Born-rule (1) takes the form

$$
p_{k}=\operatorname{tr}\left(\varrho_{\psi} \Pi_{k}\right) .
$$

We can now define the space $\mathcal{D}$ of all states to be the convex-hull of the pure states $\varrho_{\psi}$. Therefore, a general state $\varrho \in \mathcal{D}$ is a positive operator of the form

$$
\varrho=\sum_{l \in L} q_{l} \varrho_{\psi_{l}}, 0 \leq q_{l} \leq 1, \sum_{l \in L} q_{l}=1 .
$$

The numbers $q_{l}$ are empirical probabilities, which reflect ignorance of preparation, for instance. Elements of $\mathcal{D}$ are also called density-operators. The question is whether the probabilities of pure states $\varrho_{\psi}$, given by Equations (1) or (4), are any different from those in Equation (5).

As an additional tool, we need the symmetry group $U(d) \subset L\left(H_{\mathbb{C}}^{d}\right)$, consisting of the isometries of the Hilbert space $H_{\mathbb{C}}^{d}$. The action of the symmetry group on $H_{\mathbb{C}}^{d}$ leaves the scalar product $\langle\cdot \mid \cdot\rangle$ and physical quantities like average energy and entropy of systems invariant. We now have all the elements in place to recover the probabilities of pure quantum states.

\subsection{Permutation Symmetry}

The action of $U \epsilon U(d)$ on $H_{\mathbb{C}}^{d}\left|e_{k}\right\rangle \rightarrow U\left|e_{k}\right\rangle$ induces a corresponding action on the projection operators:

$$
\Pi_{k} \rightarrow \hat{\Pi}_{k}=U^{-1} \Pi_{k} U, k \leq d .
$$

It holds by Equation (2)

$$
\hat{\Pi}_{k}^{2}=U^{-1} \Pi_{k}^{2} U=U^{-1} \Pi_{k} U=\hat{\Pi}_{k} .
$$

By Equation (2), together with $U^{*}=U^{-1},\left(U^{*}\right)^{*}=U$, it holds that

$$
\hat{\Pi}_{k}^{*}=U^{*} \Pi_{k}^{*}\left(U^{-1}\right)^{*}=U^{-1} \Pi_{k}^{*} U=\hat{\Pi}_{k} .
$$

Therefore, the action maps orthogonal projectors onto themselves. In particular isometries, which map the set of basis vectors $\left\{\left|e_{k}\right\rangle\right\}_{k \leq d}$ onto itself, induce permutations on the set $\left\{\Pi_{k}\right\}_{k \leq d}$. On the other hand, for every permutation $\sigma \epsilon \Sigma(d)$ of $\left\{\Pi_{k}\right\}_{k \leq d}$ there exists $U \epsilon U(d)$ such that $U\left|e_{k}\right\rangle=\left|e_{\sigma(k)}\right\rangle$ and $\hat{\Pi}_{k}=U^{-1} \Pi_{k} U=\Pi_{\sigma(k)}$. So the permutation group of the orthogonal projectors is isomorphic to a sub-group $\Sigma(d)$ of $U(d)$. Due to the commutative property of addition, there holds for every pure state $\varrho_{\psi}$

$$
\varrho_{\psi}=\sum_{i, k=1}^{d} \psi_{i} \psi_{k}^{*}\left|e_{i}\right\rangle\left\langle e_{k}\left|=\sum_{i, k=1}^{d} \psi_{\sigma(i)} \psi_{\sigma(k)}^{*}\right| e_{\sigma(i)}\right\rangle\left\langle e_{\sigma(k)}\right|, \quad \sigma \epsilon \Sigma(d)
$$

Hence pure states are invariant under permutations $\sigma \epsilon \Sigma(d)$ of the set $\left\{\Pi_{k}\right\}_{k \leq d}$. The same holds a fortiori for the whole state space $\mathcal{D}$ as the convex-hull of the pure states.

\subsection{The Born-Rule}

Let a state $|\psi\rangle \epsilon H_{\mathbb{C}}^{d}$ be represented by $|\psi\rangle=\sum_{k=1}^{d} e^{i \varphi_{k}} \sqrt{\frac{m_{k}}{N}}\left|e_{k}\right\rangle, m_{k} \in \mathbb{N}, \varphi_{k} \in \mathbb{R}, \quad N=\sum_{k=1}^{d} m_{k}[13]$. Since the rational numbers $\mathbb{Q} \subset \mathbb{R}$ are a dense subset of the real numbers, this is general enough. We now perform, as done in [11], a generalized measurement on an ancilla-system, represented by $N$ 
orthogonal states $\left\{|n \epsilon\rangle H_{\mathbb{C}}^{N}\right\}_{1 \leq n \leq N}$. For this purpose let $M_{0}=1, M_{k}=\sum_{i=1}^{k} m_{i}, k \geq 1$ and define the set of $N$ operators $\left\{\widetilde{\Pi}_{l_{k}} \mid \widetilde{\Pi}_{l_{k}}=\frac{1}{\sqrt{m_{k}}} \Pi_{k}\right\} \quad 1 \leq k \leq d \quad$. It holds: $M_{k-1} \leq l_{k} \leq M_{k}$

$$
\sum_{n=1}^{N} \widetilde{\Pi}_{n} \widetilde{\Pi}_{n}^{*}=\sum_{k=1}^{d} \sum_{l_{k}=M_{k-1}}^{M_{k}} \widetilde{\Pi}_{l_{k}} \widetilde{\Pi}_{l_{k}}^{*}=\sum_{k=1}^{d} \Pi_{k} \Pi_{k}^{*}=\mathbb{1} .
$$

Therefore, the operators $\left\{\widetilde{\Pi}_{l_{k}}\right\} \quad 1 \leq k \leq d \quad$ define a generalized measurement, i.e., a $M_{k-1} \leq l_{k} \leq M_{k}$

unitary transformation $U$, on the combined state $|\psi\rangle \otimes|0\rangle \epsilon H_{\mathbb{C}}^{d} \otimes H_{\mathbb{C}}^{N}$

$$
|\psi\rangle \otimes|0\rangle \stackrel{U}{\rightarrow} \sum_{k=1}^{d} \sum_{l_{k}=M_{k-1}}^{M_{k}} \widetilde{\Pi}_{l_{k}}|\psi\rangle \otimes\left|l_{k}\right\rangle,
$$

or at the level of density matrices $\varrho_{\psi} \otimes|0\rangle\langle 0| \epsilon \mathcal{D} \otimes \mathcal{D}_{N}$

$$
\varrho_{\psi} \bigotimes|0\rangle\left\langle 0\left|\stackrel{u}{\rightarrow} \sum_{k=1}^{d} \sum_{l_{k}=M_{k-1}}^{M_{k}} \widetilde{\Pi}_{l_{k}} \varrho_{\psi} \widetilde{\Pi}_{l_{k}}^{*} \bigotimes\right| l_{k}\right\rangle\left\langle l_{k}\right| .
$$

Consequently, a measurement-result can be one of the $N$ states $\left\{\widetilde{\Pi}_{l_{k}} \varrho_{\psi} \widetilde{\Pi}_{l_{k}}^{*}\right\}$ $1 \leq k \leq d$
$M_{k-1} \leq l_{k} \leq M_{k}$

By permutation-symmetry of the resolution (12) and the lack of any sufficient reason to do otherwise, an observer assigns ex-ante to each possible measurement result $\widetilde{\Pi}_{l_{k}} \varrho_{\psi} \widetilde{\Pi}_{l_{k}}^{*}$ a probability $p_{l_{k}}=\frac{1}{N}$. The observer therefore holds the state

$$
\widetilde{\varrho}_{\psi}=\sum_{k=1}^{d} \sum_{l_{k}=M_{k-1}}^{M_{k}} \frac{1}{N}\left(\widetilde{\Pi}_{l_{k}} \varrho_{\psi} \widetilde{\Pi}_{l_{k}}^{*}\right) .
$$

By construction

$$
\widetilde{\varrho}_{\psi}=\sum_{n=1}^{N} \frac{1}{N}\left(\widetilde{\Pi}_{n} \varrho_{\psi} \widetilde{\Pi}_{n}^{*}\right)=\sum_{k=1}^{d} \sum_{l_{k}=M_{k-1}}^{M_{k}} \frac{1}{N}\left(\widetilde{\Pi}_{l_{k}} \varrho_{\psi} \widetilde{\Pi}_{l_{k}}^{*}\right)=\sum_{k=1}^{d} \frac{m_{k}}{N} \Pi_{k} .
$$

Since by design $\sum_{k=1}^{d} \frac{m_{k}}{N}=1$, we have due to the definition of a general state (5) and the choice of $\psi_{k}, k \leq d$,

$$
p_{k}=\frac{m_{k}}{N}=\left|\psi_{k}\right|^{2}
$$

This is the Born-rule (1), (4). Note at this point that, since the elements $\widetilde{\Pi}_{l_{k}} \varrho_{\psi} \widetilde{\Pi}_{l_{k}}^{*}$ have no mixed terms $\left|e_{i}\right\rangle\left\langle e_{k}\right|, i \neq k$, the symmetry argument in Section 2.1 holds, even if the symmetry group is reduced to a connected component $U \epsilon S U(d) \subset U(d)$, where $U\left|e_{k}\right\rangle= \pm\left|e_{\sigma(k)}\right\rangle$.

\subsection{Frequencies}

The theory so far does only cover single trials. Assume, there exists a pure state $\varrho \in \mathcal{D}$ and a complete set of projectors $\left\{\Pi_{k}\right\}_{k \leq d}$. To find probabilities for a sequence of different outcomes 
$k_{1}, \ldots, k_{N}$, of $N$ trials of an experiment on $\varrho$ we assume that the state of $\mathrm{N}$-trials $\varrho^{N}$ is given by the tensor product

$$
\varrho^{N}=\varrho \overbrace{\otimes \ldots \otimes}^{N-\text { times }} \varrho
$$

As explained in reference [14], this representation implies the independence of the single trials and we get

with probabilities

$$
p\left(k_{1}, \ldots k_{N}\right)=p_{k_{1}} \ldots p_{k_{N}}
$$

$$
p_{k}=\operatorname{tr}\left(\varrho \Pi_{k}\right) .
$$

So the outcomes of repeated measurements are identically and independently distributed (i.i.d.). The probability for outcome $k$ to occur $n_{k}$ times, $k \leq d, \sum_{k} n_{k}=N$, is given by the multinomial distribution

$$
p\left(n_{1}, \ldots n_{d}\right)=\left(N ! / n_{1} ! \ldots n_{d} !\right) p_{1}^{n_{1}} \ldots p_{d}^{n_{d}} .
$$

The individual counting functions $n_{k}$ are binomially distributed and hence $E\left(n_{k}\right)=N p_{k}$. For large $N$ the averages, $\bar{n}_{k}$, of the statistical counting functions approach the expectation values and therefore

$$
\bar{n}_{k} / N \approx p_{k} .
$$

The fact that $\bar{n}_{k} \rightarrow E\left(n_{k}\right), N \rightarrow \infty$, is due to the law of large numbers. The frequencies indeed replicate the probabilities. This is achieved by the independence-assumption for the multi-trial states $\varrho^{N}, N \epsilon \mathbb{N}$, reflected in Equations (17), (19) and (20). All the probabilities involved can be interpreted classically.

\section{The Notion of "Time"}

In the above paragraphs we were able to introduce the proabilities of pure quantum states as a result of unitary symmetry. To achieve this, we made use of the permutations, which form a subgroup of the symmetry group $\Sigma(d) \subset U(d)$. Where does quantum physics need the full symmetry group?

\subsection{Identity and Change}

So far we considered quantum physics in a purely static way. There are two fundamental intuitions about the world, which in combination force us to extend the model. The first one is the intuition of "identity". Some physical system can be recognized as being individual and hence as having an identity. (Of course, quantum physics knows systems, like highly entangled ones or elements of Fock-space, which lack individuality.) In this paragraph we do not work in the mathematical model of quantum physics but with abstract functional relations. Let $I \epsilon \Lambda$ be an element of the set of individual systems. We assume that such an individual system is uniquely defined by all its properties (here a "property" can be anything, which uniquely defines the system) i.e., $I=I(\bar{a})$, where $\bar{a} \in A$ denotes an element of a set of properties $A$. So we have a one-to-one correspondence $\bar{a} \leftrightarrow$ I between the set of properties and the set of individual systems. The second intuition is that a system can have different properties without losing its identity, i.e., $J(\bar{a})=I(\bar{b})$, even if $\bar{a} \neq \bar{b}$. Because of the one-to-one relationship $\bar{a} \leftrightarrow \mathrm{I}$, this is logically only possible by the introduction of an extra degree of freedom $\tau \epsilon T$, such that the map $A \times T \rightarrow \Lambda:(\bar{a}, \tau) \mapsto J(\bar{a}, \tau)$ satisfies

$$
\left(\exists\left(\bar{a}, \tau_{1}\right)=\Xi\left(\bar{b}, \tau_{2}\right)\right) \wedge(\bar{a} \neq \bar{b}) \Rightarrow \tau_{1} \neq \tau_{2} .
$$

Relation (21) implies that the restriction of the map to fixed $\tau$ is one-to-one, whereas the whole map is not. We call this degree of freedom $\tau \epsilon T$ "time". "Time" is by construction not on equal footing with the properties $\bar{a}$. At a given $\tau$ a system has exactly one set of properties $\bar{a}$, which can, however, 
vary with $\tau$ without violating identity. This is what we call "change". Given the above, we can represent the system $\Xi(\bar{a}, \tau)$ simply by a map $T \rightarrow A, \tau \mapsto \bar{a}_{\tau}$, where $\tau$ labels the defining properties $\bar{a}$ at "time-point" $\tau$. We see that there is a one-to-one correspondence between any two "time-points" $\bar{a}_{\tau_{1}} \leftrightarrow \bar{a}_{\tau_{2}}$ in order to preserve identity.

Our arguments have so far been logical by nature, and we now need to come back to the model of quantum physics, which we introduced in Section 2, and to give the abstract notions $\bar{a}$ and $\tau$ a representation in the model.

\subsection{Dynamics}

We have seen that in quantum physics an observable is represented by a self-adjoint operator $A \epsilon L\left(H_{\mathbb{C}}^{d}\right)$ over the Hilbert space of states $|\psi \epsilon\rangle H_{\mathbb{C}}^{d}$. A system $|\psi\rangle$ is hence uniquely defined by the set of coefficients $\left\{\left.c_{i}^{A}\left|c_{i}^{A} \in \mathbb{C}, \sum_{i=1}^{d}\right| c_{i}^{A}\right|^{2}=1,1 \leq i \leq d\right\}$ of its resolution in the eigenbasis of $A$. As we have seen in the first part of the paper, the coefficients have a natural interpretation as probabilities $p_{i}=c_{i}^{A}\left(c_{i}^{A}\right)^{*}=\left|c_{i}^{A}\right|^{2}$. With respect to the basis of a different self-adjoint operator $B \in L\left(H_{\mathbb{C}}^{d}\right)$, there is an element $U \epsilon U(d)$, such that for the coefficients with respect to $B, c_{i}^{B} \in \mathbb{C}, 1 \leq i \leq d$, there holds $c_{i}^{B}=\sum_{k=1}^{d} U_{i k} c_{k}^{A}$. This holds because both operators have an orthonormal eigenbasis and total probability has to be preserved. So in the terminology of Section 3.1, we can identify the system uniquely by the vector $\vec{c}^{A}=\left(c_{1}^{A}, \ldots, c_{d}^{A}\right) \in \mathbb{C}^{d}$, and the coefficients $c_{i}^{A} \in \mathbb{C}, i \leq d$, correspond to the properties. The elements $U \epsilon U(d)$ relate the properties and form equivalence-classes of vectors representing the same system.

The degree of freedom $\tau$ is represented by the real numbers $t \in \mathbb{R}$. This choice is close to our intuition of an ordering between time-points " $s<t$ " and of the continuity of "change". If time is quantized, then $n \in \mathbb{Z}$ might be a more appropriate choice, but as we will see, a quantization of some kind is also implied by the choice of $t \in \mathbb{R}$. As noted in Section 3.1, a necessary condition for two different vectors $\overrightarrow{c_{t_{1}}^{A}}$ and $\overrightarrow{c_{t_{2}}^{A}}$ to represent the same system is, that there exists a one-to-one correspondence between the two under preservation of total probability. Hence we are led to the symmetry-group $U(d)$ and demand that there is $U\left(t_{2}, t_{1}\right) \epsilon U(d)$ such that $\overrightarrow{c_{t_{2}}^{A}}=U\left(t_{2}, t_{1}\right) \overrightarrow{c_{t_{1}}^{A}}$. Since we chose the time-parameter to be continuous $t \in \mathbb{R}$, we can think of a curve or trajectory through $\mathbb{C}^{d}$ and write

$$
\overrightarrow{c_{t}^{A}}=U(t) \overrightarrow{c_{0}^{A}}, U(0)=\lessdot
$$

Note that the relation $\overrightarrow{c_{t_{2}}^{A}}=U\left(t_{2}, t_{1}\right) \overrightarrow{c_{t_{1}}^{A}}$ is symmetric in time, i.e., $\overrightarrow{c_{t_{1}}^{A}}=U^{*}\left(t_{1}, t_{2}\right) \overrightarrow{c_{t_{2}}^{A}}$.

\subsection{Quanta of Time}

"Time" was introduced in Section 3.1 to allow variance of properties under preservation of identity. This is what we called "change". "Time" is hence a different kind of degree of freedom than those encoded in the vector $\vec{c} \epsilon \mathbb{C}^{d}$, and we cannot expect that there is an operator, which measures "time". (There is an argument of W. Pauli: If $T$ were a self-adjoint time-operator conjugate to $H$, then $[T H]=i \hbar$ and the energy spectrum would be unbounded.) In fact, one point $t_{0}$ of any trajectory can be fixed arbitrarily; hence, it has the aspect of a gauge, and we can assume that $t_{0}=0$. The duration $\Delta t=\left(t-t_{0}\right)=t$, however, is relational and indeed a measure of change. If a system is in the state $\overrightarrow{c_{t}}=U(t) \overrightarrow{c_{0}}$, then the probability that a measurement finds it in the state $\overrightarrow{c_{0}}$ is $\left|\left\langle\overrightarrow{c_{t}} \mid \overrightarrow{c_{0}}\right\rangle\right|^{2}$; hence, the probability that $\overrightarrow{c_{0}}$ has undergone change after a time-interval of $t$ is

$$
P(t)=1-\left|\left\langle\overrightarrow{c_{t}} \mid \overrightarrow{c_{0}}\right\rangle\right|^{2}
$$


In particular, if $\overrightarrow{c_{t}} \perp \overrightarrow{c_{0}}$, then $P(t)=1$ and there is certain change. By Equation (23) we can predict the likelihood that an initial state $\overrightarrow{c_{0}}$ is in a different state $\overrightarrow{c_{t}}$ after time $t$, if we know the evolution operator $U(t), t \geq 0$. $U(t)$ can be written

$$
U(t)=e^{-\frac{i}{\hbar} H t}, t \geq 0 .
$$

$H \epsilon L\left(H_{\mathbb{C}}^{d}\right)$ denotes a self-adjoint operator representing the observable "energy". (We assume the operator $\mathrm{H}$ to be constant.) So $U(t), t \geq 0$ is known, if $H$ is, and the potential for change is an observable of the system. With $\bar{E}$ denoting the average energy $\bar{E}=\left\langle\overrightarrow{c_{0}}|H| \overrightarrow{c_{0}}\right\rangle$ and $E_{0}$ the lowest eigenvalue of $H$, it holds by a theorem in reference [15] that the minimum time needed to reach an orthogonal $\overrightarrow{c_{t}}$, and hence certain, change, is

$$
t_{\min }=\frac{h}{4\left(\bar{E}-E_{0}\right)} .
$$

Expression (25) defines a natural time scale by $d t^{\prime}=\frac{1}{t_{\min }} d t$. If an evolution is well behaved, like in a measurement for instance, we may assume that the function $P:\left[0, t_{\min }\right] \rightarrow[0,1]$ in Equation (23) is monotonous. So not only the potential for change but also the time-quantum for certain change is embodied in the energy operator $H$. It is in this sense that time in quantum physics is quantized, and $t_{\min }$ is a "quantum of certainty". We call theories, in which change is certain for every $t>0$, temporally deterministic.

"Time", introduced in Section 3.1, is clearly a real and by Equation (21) logically necessary degree of freedom of any physical system, keeping its identity under change. But does the parameter " $t \in \mathbb{R}^{\text {" }}$ really represent what we experience as "time"? As mentined before, there is no operator, which could measure the parameter " $t$ ". In addition " $t$ " is system-dependent, and we never measure the vector $\overrightarrow{c_{t}}$. What we measure are elements $\lambda \in \mathbb{R}$ of the the spectrum $\sigma_{A}$ of an observable $A$, and by doing so we break the symmetry (22). In addition, the time-parameter " $t$ " does not distinguish between past and future, since Equation (23) is invariant under time-reversal $t \rightarrow-t$. We will now show how breaking the symmetry (22) can lead to a flow, which carries qualities of empirical time.

\subsection{Thermal Flow}

Assume that an observable is measured on a system $\overrightarrow{c_{0}}=\sum_{i \in I} c_{i}\left|e_{i}\right\rangle$ by an apparatus $\mathcal{A}$ with pointer-states $\left\{A_{i}\right\}_{i \in I}$ such that the joint system after measurement-interaction is represented by $|\psi\rangle=\sum_{i \in I}\left\langle\overrightarrow{c_{0}} \mid i\right\rangle|i\rangle$, where $|i\rangle=\left|e_{i}\right\rangle \otimes\left|A_{i}\right\rangle$ and $\langle i \mid j\rangle=\delta_{i j}$. If $\varrho=|\psi\rangle\langle\psi|$ is the corresponding density matrix, then there is entropy $S_{\varrho}=-\operatorname{tr}\left(\rho_{\log } \varrho\right)$, encoded in $\overrightarrow{c_{0}}$. The reduction to a single outcome breaks the symmetry (22) and reduces the entropy $S_{\varrho}$ to zero. By the second law, there is minimally the equal amount of entropy dissipated to the environment $\left|\varepsilon_{0}\right\rangle$. If the system is in an environmental heat-bath at temperature $T$, then there is consequently a (minimal) average dissipation of energy to the environment of the amount $\bar{E}=S_{\varrho} k_{B} T=E_{k_{B} T \log _{2} \varrho}(\varrho)$, where $k_{B}$ is the Boltzmann constant. The origin of the dissipation is the erasure of a former, unknown apparatus-system state, a process, which is irreversible [16]. The induced interaction with the environment can be assumed to leave the system-apparatus state $|\psi\rangle$ invariant

$$
\left|\varepsilon_{0}\right\rangle=\sum_{i \in I}\left\langle\overrightarrow{c_{0}} \mid i\right\rangle|i\rangle\left|\varepsilon_{0}\right\rangle \stackrel{U_{0}^{t}}{\rightarrow} \sum_{i \in I}\left\langle\overrightarrow{c_{0}} \mid i\right\rangle|i\rangle\left|\varepsilon_{i}\right\rangle=\left|\varepsilon_{1}\right\rangle
$$

There holds $\left\langle\varepsilon_{0} \mid \varepsilon_{i}\right\rangle=0$, i $\epsilon$, and hence $\left\langle\varepsilon_{0} \mid \varepsilon_{1}\right\rangle=0$. By Equation (25) there results a time-scale

$$
t_{\min }^{\varrho}=\frac{h}{4 S_{\varrho} k_{B} T} .
$$


After $t_{\text {min }}^{\varrho}$ the environment "knows" with certainty that a measurement has happened. Note the similarity with the thermal time-flow defined in [17], where the flow is introduced in an abstract operator-algebra. A thermal clock hence divides time into steps of

$$
d \tau=\frac{1}{t_{\min }^{\varrho}} d t=\frac{4 k_{B} T S_{\varrho}}{h} d t
$$

The thermal flow is still system-dependent, but it has an implicit direction due to the irreversibility of its origin, namely erasure. Of course, since we fall back to quantum theory to develop Equation (27), we cannot expect an explicit asymmetry. There is again no direct measurement of thermal-time in the sense of observables. It can in principle be measured, if the $\left|\varepsilon_{i}\right\rangle, i \in I$, are pointer-like states. This way the environment serves as a clock. The thermal flow is fuelled by the entropy of a system and the thermal energy of the heat-bath. The system-dependence is not an obstacle, since we can gauge two clocks, which evolve with time-parameters $\tau_{1}$ and $\tau_{2}$, say, by demanding them to march in step. This means that there is $\alpha \in \mathbb{R}, \alpha>0$, such that

$$
d \tau_{1}=\alpha d \tau_{2}
$$

So any physical system, whose evolution develops linearly $(\alpha>0)$ with a thermal flow, can be called a thermal clock. If we set $\alpha=\frac{1}{S_{e}}$ we have

$$
d \tau=\frac{\alpha}{t_{\min }^{\varrho}} d t=\frac{4 k_{B} T}{h} d t
$$

In this sense there exists a universal thermal clock. Gauging the universal thermal clock with other clocks, like oscillating photons for instance, leads to various interesting results in the geometry of space-time, in particular to the derivation of Einstein's equations. Related work can be found in references $[18,19]$.

We do not know for sure whether unitary symmetry-breaking and the corresponding thermal time-flow are ontological or merely perspectival. A deeper discussion is found in, e.g., reference [20]. In this paper we treat it as a fact, which offers an explanation as to why empirical time is directed. This has the further consequence that, along the Feynman-Stückelberg interpretation, anti-matter cannot last in empirical space-time. The mechanism in Section 3.4 also offers an interpretation of the Big Bang. The first collapse in a coherent universe was the beginning of empirical time and must have caused en enormous energy dissipation, if we assume that the average energy per bit of information $T=\frac{\partial E}{\partial S}$ was very large. Yet, our theories of nature are only aware of a symmetric time parameter. We want to dicuss in the next paragraph to what degree these theories can coexist with the intuition of empirical time and the thermal flow.

\section{Space-Time}

Let the observable $A \in L\left(H_{\mathbb{C}}\right)$ be "position in physical space". The corresponding vectors $\overrightarrow{c_{x}}, x \in \mathbb{R}^{3}$, representing a single quantum system, are no longer elements of a finite-dimensional but of an infinite-dimensional Hilbert space $\overrightarrow{c_{x}} \in L_{\mathbb{C}}^{2}\left(\mathbb{R}^{3}\right)$. Quantum physics does not describe "change" $\overrightarrow{c_{t}}(x)$ directly in physical space, and the time-parameter, with respect to which it is symmetric, does not reflect the empirical time of a dynamically developing "present". It is, however, able to give an account of this experience by collapse and the corresponding erasure of a former "present". This process induces the thermal time-flow (26). The theories, which describe change directly in physical space, are the classical ones, including relativity theory. But they also are invariant with respect to time-reversal. In addition, there is a sort of incompatibility between the notion of collapse and relativity, since a collapse allows the definition of a preferred reference frame. In summary we are left with the following situation: Either our theories of nature live outside of empirical space-time, like quantum physics, or 
they live in empirical space, are deterministic and their time-parameter lives outside empirical time. In addition, the way that quantum physics offers to reconstruct our empirical space-time seems at odds with relativity. It looks as if theory and experience of space and time live in different "worlds", and our narratives sometimes confuse them.

\subsection{Speed of Light}

Special relativity is a classical theory, where change of position is temporally deterministic. It is characterized by two "axioms": (1) The independence of the speed of light in vacuum with respect to inertial frames; (2) The covariance of all physical laws with respect to the respective symmetry-transformations, the elements of the Lorentz group. If we characterize the speed of light, $c$, locally as the maximum possible velocity of any freely moving physical system $|\psi\rangle$, it must be invariant with respect to relative motion. Since change is temporally deterministic, relation (25) suggests that there can be no instantaneous change of position. Therefore, if a system "moves" a distance $d$, then we have for its average velocity $\bar{v}_{|\psi\rangle} \leq \frac{d}{t_{\min }}<\infty$. This fits well into Newtonian physics, where velocities are finite but can still be arbitrarily large. We have to explain why it holds $\bar{v}_{|\psi\rangle} \leq c$. The concept of thermal time allows us to sketch a possible solution. Assume that we are in an expanding de-Sitter universe with a cosmic horizon at radius $R_{\infty}$ from an inertial observer. With $a_{\infty}$ denoting the acceleration of the horizon and $H_{0}$ the Hubble constant, it holds

$$
a_{\infty}=c H_{0}=\frac{c^{2}}{R_{\infty}}
$$

An inertial observer measures thermal radiation of temperature $T_{\infty}$ [21], which allows the definition of a thermal flow. By Equation (30), we get for the average velocity of any physical system, locally "moving" over a distance $d$,

$$
\bar{v}_{|\psi\rangle} \leq \frac{d}{t_{\min }}=\frac{4 k_{B} T_{\infty} d}{h} .
$$

For the Unruh-de-Sitter temperature $T_{\infty}=\frac{\hbar a_{\infty}}{2 \pi k_{B} C}$ we have by Equation (31)

$$
T_{\infty}=\frac{\hbar a_{\infty}}{2 \pi k_{B} c}=\frac{\hbar H_{0}}{2 \pi k_{B}}
$$

Since $H_{0}=\frac{c}{R_{\infty}}$ and $d_{\infty}=\frac{\pi}{2} R_{\infty}$, Equation (32) turns into

$$
\bar{v}_{|\psi\rangle} \leq \frac{4 k_{B} T_{\infty} d}{h}=\frac{4 k_{B}}{h} \cdot \frac{\hbar c}{2 \pi k_{B}} \cdot \frac{\pi d}{2 d_{\infty}} \leq c .
$$

Measuring time by the quanta of the thermal clock of an expanding de-Sitter vacuum locally limits average velocities of material systems. It is natural to expect a model of empirical time to play a role in an explanation of the limit (34), since we measure the speed of light by experiments in empirical space-time, after all. So the first postulate of relativity coexists consistently with quantum physics and empirical space-time. The situation with covariance turns out to be more intricate.

\subsection{Covariance}

If probabilities are to be independent of inertial reference frames, then the probabilities of two space-like separated measurements have to be independent of their time-order. Let us consider the probabilities of two spin-measurements $A, B \epsilon\{ \pm 1\}$ on a pair of entangled photons in freely chosen directions $a, b$. We denote the outcomes by $\mathcal{A}=(A, a)$ and $\mathcal{B}=(B, b)$. In the sequel we follow an argument in references [22,23], and the formulation is independent of the details of quantum theory. Assume that the measurement $\mathcal{A}$ happens before $\mathcal{B}$ and that there is no influence from the future, what 
we call no "retrocausality". (The notion of "causation" is intricate and we use the word simply to express the independence of correlations from future events.) This means that events in the future, in particular $\mathcal{B}$, have no impact on the probabilities of $\mathcal{A}$. Denote by $\lambda_{\mathcal{A}}$ all the variables, which could otherwise influence $\mathcal{A}$ independently of $a$. Since $\mathcal{A}$ happens first, and there is no retrocausality, we have

$$
P_{\mathcal{A}}\left(A \mid a, B, b, \lambda_{\mathcal{A}}\right)=P_{\mathcal{A}}\left(A \mid a, \lambda_{\mathcal{A}}\right) .
$$

The analogous conclusion holds for a reference frame, where $\mathcal{B}$ happens before $\mathcal{A}$

$$
P_{\mathcal{B}}\left(B \mid b, A, a, \lambda_{\mathcal{B}}\right)=P_{\mathcal{B}}\left(B \mid b, \lambda_{\mathcal{B}}\right)
$$

Independence of $P_{\mathcal{A} / \mathcal{B}}$ of time order together with Equation (35), Equation (36) and $\lambda=\lambda_{\mathcal{A}} \cup \lambda_{\mathcal{B}}$, define a Bell-local model

$$
P(A B \mid a, b, \lambda)=P_{\mathcal{A}}(A \mid B, a, b, \lambda) \cdot P_{\mathcal{B}}(B \mid a, b, \lambda)=P_{\mathcal{A}}(A \mid a, \lambda) \cdot P_{\mathcal{B}}(B \mid b, \lambda) .
$$

Such models contradict quantum physics theoretically [24] and experimentally [25]. As a consequence, there are either preferential reference frames, contrary to relativity, or we have to drop the assumption of no retrocausality. There has been renewed interest in the question of retrocausality [26-28], and its existence seems, not least given the above result, a sensible way within a realist interpretation of quantum physics to avoid serious tension with relativity. (There are other possibilities, like renouncing the causal Markov property, which prevents the first factorization in Equation (37), or assuming a kind of gigantic conspiracy, which prevents the free setting of $a$ and $b$. We could also think of the two entangled photons in three space-dimensions as of one in six dimensions and thus overcome non-locality. In case we do not attest to any of the notions in quantum physics to represent something real and take a purely epistemic view instead, then the above arguments are obsolete.) It is, however, evident that the picture of empirical space-time, which we introduced in Section 3.4, where the "present" dynamically emerges, cannot be made compatible with retrocausality, not even in its temporally non-local form. So, again, we have to either accept a violation of a postulate of relativity or accept the reality of a realm, maybe parallel to empirical space-time, where correlations can depend on past and future events. In any case we cannot have it all in one, i.e., a relativistic quantum theory in empirical space-time. This is the key conclusion.

\section{Consequences}

We have shown that the notions of unitary symmetry and symmetry-breaking are central to the understanding of probabilities and "change" in quantum physics. Of course, symmetry-preservation and symmetry-breaking, respectively, lead to different regimes and it seems that the theory of relativity, in particular, combines aspects of both. The tensions between relativity and quantum physics are thus based on the insufficient distinction between two realms. Some conundrums in quantum physics, like the parallel existence of collapse and the unitary evolution of a realist wave function [29], or the quantum liar paradox and others [30,31], can be better understood under the assumption that quantum physics does not live in empirical space-time. There is work which recognizes the existence of two realms as a possible way forward [32,33]. The success of the thermal time-flow to explain phenomena in general relativity $[18,19]$ and the arguments in Sections 3 and 4 of this paper also suggest that the assumption of the existence of two realms, which influence each other in a specific way, is worth further exploration. Ultimately, falling back again on our human experience, is it not the case that our mind constantly anticipates future possibilities, which do not yet exist in physical space-time but influence our actions in the present, which in turn help shape the future? Maybe we should take our experience seriously and try to shape our theories of nature accordingly.

Funding: This research received no external funding. 
Conflicts of Interest: The authors declare no conflict of interest.

\section{References}

1. Lewis, P.J. Phenomena and Theory. In Quantum Ontology, 1st ed.; Oxford University Press: New York, NY, USA, 2016; pp. 22-24, ISBN 978-0190469818.

2. Weinberg, S. Symmetry: A “Key to Nature's Secrets". The New York Review of Books, October 2011.

3. Childers, T. Philosophy E Probability, 1st ed.; Oxford University Press: New York, NY, USA, 2013; ISBN 978-0199661831.

4. Gleason, A.M. Measures on the closed subspaces of a Hilbert space. J. Math. Mech. 1957, 6, 885-893. [CrossRef]

5. Bohr, A.; Ulfbeck, O. Genuine Fortuitousness: Where Did That Click Come From? Found. Phys. 2001, 31, 757-774. [CrossRef]

6. Caves, M.C.; Fuchs, C.A.; Schack, R. Subjective probability and quantum certainty. Stud. Hist. Philos. Sci. Part B 2007, 38, 255-274. [CrossRef]

7. Saunders, S.; Barrett, J.; Kent, A.; Wallace, D. (Eds.) Many Worlds? 1st ed.; Oxford University Press: Oxford, UK, 2010; ISBN 978-0-19-956056-1.

8. Albert, D.; Loewer, B. Interpreting the many-worlds interpretation. Synthese 1988, 77, 195-213. [CrossRef]

9. Hardy, L. Quantum Theory from Five Reasonable Axioms. arXiv, 2001, arXiv:quant-ph/0101012.

10. Schack, R. Quantum Theory from Four of Hardy's Axioms. Found. Phys. 2003, 10, 1461-1468. [CrossRef]

11. Schlatter, A. Quantum Probabilities and Maximum Entropy. Entropy 2017, 19, 304. [CrossRef]

12. Born, M. Zur Quantenmechanik der Stoßvorgänge. Zeitschrift für Physik 1926, 37, 863-867. [CrossRef]

13. Zurek, W.H. Environment-assisted invariance, entanglement and probabilities in quantum physics. Phys. Rev. Lett. 2003, 90, 120404. [CrossRef]

14. Caves, C.M.; Fuchs, C.A.; Schack, R. Quantum probabilities as Bayesian probabilities. Phys. Rev. A 2002, 65, 022305. [CrossRef]

15. Margolus, N.; Levitin, L.B. The maximum speed of dynamical evolution. Physica D 1998, 120, $188-195$. [CrossRef]

16. Lubkin, E. Keeping the Entropy of Measurement: Szilard revisited. Int. J. Theor. Phys. 1987, 26, 523-534. [CrossRef]

17. Connes, A.; Rovelli, C. Von Neumann Algebra Automorphisms and Time-Thermodynamics Relation in Generally Covariant Quantum Theories. Class. Quant. Grav. 1994, 11, 2899-2917. [CrossRef]

18. Martinetti, P.; Rovelli, C. Diamond's Temperature: Unruh effect for bounded trajectories and thermal time hypothesis. Class. Quant. Grav. 2003, 20, 4919-4932. [CrossRef]

19. Schlatter, A. On the Principle of Synchronization. Entropy 2018, 20, 741. [CrossRef]

20. Kastner, R.E. On Quantum Collapse as a Basis for the Second Law of Thermodynamics. Entropy 2017, 19, 106. [CrossRef]

21. Gibbons, G.; Hawking, S. Cosmological Event Horizons, Thermodynamics, and Particle Creation. Phys. Rev. D 1977, 15, 2738-2751. [CrossRef]

22. Gisin, A. Impossibility of covariant, deterministic non local hidden-variable extensions of quantum theory. Phys. Rev. A 2011, 83, 020102. [CrossRef]

23. Dürr, D.; Lazarovici, D. Nichtlokalität durch Retrokausalität. In Verständliche Quantenmechanik, 1st ed.; Springer Spektrum, Springer-Verlag GmbH: Heidelberg, Germany, 2018; pp. 215-217, ISBN 978-3-662-55888-1.

24. Bell, J.S. La Nouvelle cuisine. In Speakable and Unspeakable in Quantum Mechanics; Cambridge University Press: Cambridge, UK, 2004; pp. 232-248, ISBN 9780521523387.

25. Hensen, B.; Bernien, H.; Dréau, A.E.; Reiserer, A.; Kalb, N.; Blok, M.S.; Ruitenberg, J.; Vermeulen, R.F.; Schouten, R.N.; Abellán, C.; et al. Loophole-free Bell inequality violation using electron spins separated by $1.3 \mathrm{~km}$. Nature 2015, 526, 682-686. [CrossRef]

26. Leifer, M.S.; Pusey, M.F. Is a time symmetric interpretation of quantum theory possible without retrocausality? Proc. R. Soc. Lond. A Math. Phys. Eng. Sci. 2017, 473, 20160607. [CrossRef] [PubMed]

27. Price, H. Toy models for retrocausality. Stud. Hist. Philos. Mod. Phys. 2008, 39, 752-761. [CrossRef]

28. Adlam, E. Spooky Action at a Temporal Distance. Entropy 2018, 20, 41. [CrossRef] 
29. Maudlin, T. Three measurement problems. Topoi 1995, 14, 7-15. [CrossRef]

30. Elitzur, A.C.; Dolev, S.; Zeilinger, A. Time reversed epr and the choice of histories in quantum mechanics. In Proceedings of XXII Solvay Conference in Physics. Special Issue, Quantum Computers and Computing; World Scientific: London, UK, 2002; pp. 452-461.

31. Aharonov, Y.; Bergmann, P.G.; Lebowitz, J.L. Time Symmetry in the Quantum Process of Measurement. Phys. Rev. B 1964, 134, 1410. [CrossRef]

32. Kastner, R.E. The Possibilist Transactional Interpretation and Relativity. Found. Phys. 2012, 42, 1094-1113. [CrossRef]

33. Elitzur, A.C.; Dolev, S. Becoming as a Bridge between Quantum Mechanics and Relativity. In Endophysics, Time, Quantum and the Subjective; Saniga, M., Buccheri, R., Elitzur, A.C., Eds.; World Scientific Publishing Co.: Singapore, 2005; pp. 197-201.

(C) 2018 by the author. Licensee MDPI, Basel, Switzerland. This article is an open access article distributed under the terms and conditions of the Creative Commons Attribution (CC BY) license (http:/ / creativecommons.org/licenses/by/4.0/). 